\title{
Huygens on Inertial Structure and Relativity
}

\author{
Marius $\operatorname{Stan}^{*+}$
}

I explain and assess here Huygens's concept of relative motion. I show that it allows him to ground most of the Law of Inertia and also to explain rotation. Thereby his concept obviates the need for Newton's absolute space. Thus, his account is a powerful foundation for mechanics, although not without some tension.

1. Introduction. While Huygens's exegetes, few as they are, agree that his mechanical foundations are profound and important, their consensus still relies too much on hagiographic pieties instead of facts. It is because their evidential basis was always partial and now is badly outdated. Predictably, this drawback has allowed some to doubt Huygens's depth of insight (Earman 1989, 70).

I set out here to redress these defects by way of a new account of his doctrine of relative motion and its role in grounding mechanics. For that, I draw on Codex Huygens $7 A$, a complete record of his extant thoughts on relativity. ${ }^{1}$ And, I rely on two hermeneutic principles. One is Contextualism:

Received October 2014; revised July 2015.

* To contact the author, please write to: 357 Stokes North, Boston College, Chestnut Hill, MA 02467; e-mail: marius.c.stan@gmail.com.

$\dagger$ For deeply illuminating exchanges, I am greatly indebted to Nick Huggett, Katherine Brading, and Robert Rynasiewicz. For stimulating, constructive discussions, I am grateful to Alan Love, George E. Smith, Peter Distelzweig, David Marshall Miller, Ed Slowik, and Victor Boantza. For helpful feedback, I thank audiences at the University of Minnesota and the Max Planck Institute for History of Science.

1. The Codex is a set of fragments in Latin and French, many on the nature of motion. They range in date from 1686 to about 1694. Huygens wrote them as drafts of a long preface to a tract in mechanics that he died without writing. I cite them by fragment and section (e.g., 9.a) from the critical edition in Mormino (1993) and Huygens's Oeuvres complètes (1888-1950) by volume and page number, e.g., 16:461. All translations are mine. So is all emphasis in citations.

Philosophy of Science, 83 (April 2016) pp. 277-298. 0031-8248/2016/8302-0007\$10.00

Copyright 2016 by the Philosophy of Science Association. All rights reserved. 
explaining an author's view from the science of her time, her aims, and in terms available then-not from the viewpoint of current physics. Garber (1992) and Friedman (2013) have applied it, respectively, to Descartes and Kant with splendid results, so I emulate them here. The other is Charity: if an author of proven deep physical insight is silent on an elementary point, she may be credited with it. Charity is justified prima facie, so the burden of argument lies with its opponents. In general, we must keep in mind that Codex $7 A$ consists in unpublished notes, inchoate to various degrees. Even so, the deep ideas they contain are well worth our attention.

First I elucidate Huygens's term 'relative motion' and locate it in the taxonomy of early modern positions (secs. 3 and 4). Then I explain its dual grounding role for mechanics: it allows him to retrieve the correct empirical content of the Law of Inertia, minus chronometry, and to account for the dynamically distinguished phenomena of rotation, by showing them to be relative motions (secs. 5 and 6). Flanking my account on either side are critical engagements with previous interpretations.

2. The Received View. After Lange in 1886 piqued modern curiosity in Huygens's theory of motion, Stein made it part of a historiographical arc bending toward the general theory of relativity. His interest was in Huygens's view that motion is relative, which he construed as "(absolute) difference-of-velocities," namely, of four-velocities in Newtonian spacetime. As this "remarkable insight" was beyond the expressive ken of early modern terms, Stein excused as "paradoxical" Huygens's official account of relativity $(1977,7-10)$.

Earman too saw in Huygens a contributor to the long debate on absolute and relative motion. As a first step toward resolution, Earman explicated 'relative' as the conjunction of two theses. R1: All motion is the relative motion of bodies. R2: Spatial and temporal relations among bodies and events are direct. He took Huygens to subscribe to both but remained wary of Stein's laudatory account of Huygens on relativity (Earman 1989, 12, 42-43, 67ff.).

In the same decade, Barbour praised Huygens for "looking forward to Einstein" and for his success in "separating absolutely cleanly two components" of motion, inertial and accelerated. Still, Barbour complained, Huygens remained "quietly discreet" about the first component, in that he failed to explain "with respect to what" inertial paths are straight lines $(2001,495$, 675; his italics).

These influential readings suffer from some common defects. First, they are too cursory to do justice to Huygens's full, considered view-whose rich sophistication they miss, working as they do with partial, selective evidence. Second, their key premise is flawed: allegedly, the problem is whether motion is absolute or relative. But they leave 'absolute' undefined, 
use 'relative' equivocally, and fail to show that their problem was Huygens's too. Third, their methodology is suspect: often they refuse to take Huygens at face value, opting instead to inflict on him the anachronistic vocabulary of differential geometry. My interpretation avoids these shortcomings - to what extent, I try to judge in section 7.

3. Motion: True, Absolute, Relative. Huygens's interpreters and historically minded philosophers of physics share the presumption that his basic problem was whether motion is absolute or relative. Thinking that the question is univocal, they expect it to have a clear answer. But, they miss that 'absolute' and 'relative' were equivocal in early modern times, so they need careful disentangling or else the problem of motion is not well posed. Here is an attempt. ${ }^{2}$

In one sense, 'absolute motion' was just a synonym for the equivalent phrases, 'real', 'proper', 'physical', and 'true motion'. Hence, 'motion is absolute' was the thesis that any body has a true $=$ real $=$ proper $=$ physical motion or rest, expressed by a determinate quantity - a privileged velocity and that there is a fact about whether the body moves or rests. Thus, motion is a complete predicate, not an irreducible relation. Call this view Completism.

In another sense, 'absolute' denoted an explanatory commitment. 'Motion is absolute' meant that true motion consists in change of place in absolute space. Call this view Absolutism.

'Relative' was so ambiguous that it shows up in incompatible views. In one sense, it denoted an explanatory commitment. 'Motion is relative' meant that true motion consists in kinematic change - actual or just latentrelative to some preferred material frame: the earth, the stars, the body's material envelope, the mass center of interaction, and so on. Call this view T-Relationism. ${ }^{3}$

In another sense, 'relative motion' signaled the denial of Completism. Thus, 'motion is relative' meant that bodies have no true $=$ absolute $=$ proper $=$ real motions. Call this view Relativism.

The entailment relations between these views are as follows. Absolutism and T-Relationism are contrary explanations of Completism. Relativism is the negation of Completism and entails that both Absolutism and T-Relationism are false. Ergo, T-Relationism and Relativism are logically incompatible - a crucial reason to be careful about what early modern figures, especially Huygens, mean by 'relative motion'. Now for some direct evidence.

2. I build here on an insight from Rynasiewicz (2000), which I regard as fundamentally correct.

3. I thank Nick Huggett for this helpful suggestion. 
Completism was the view of a heavy majority and the default position in astronomy. Riccioli, the last doyen of the Ptolemaic ancien régime, reveals in Almagestum novum that both sides in the Copernican debate shared it:

In the Ptolemaic system, because the motion of the superior [planets] is hampered by the Epicycles ... sometimes they truly [reipsa] do not move at all, as far as their true motion [motum verum] under the Fixed stars is concerned - but appear to stay still [stare videntur]; whereas sometimes they retrogress westward. However, in the Copernican hypothesis this Phenomenon is just an appearance [apparentia], resulting not from the object itself, i.e. from the real motion [motus realis] of the Planets, but from the translation of our eyes together with the Earth. (Riccioli 1651, 302)

Ismael Boulliau also conceded Completism: "We judge rest to be true as well as apparent" $(1645,101)$. Likewise, a renegade Copernican: "I grant that, to our eyes, it appears that the Earth rests and the Sun moves in the sky. However, in reality [revera] this is not so" (Lansberg 1651, preface). Horrocks as well: "[Copernicus] infers correctly that the places of the Fixed stars, which are in fact unmoved [revera], only seem to the eye and the imagination to lag behind [the Zodiac]. Kepler assents to this view. Lansberg too was once of the right opinion in this matter-i.e. that the fixed stars really are fixed [re immotas], as their name would have it. Later he changed his mind, attributing to the stars a very slow motion" $(1678,54)$. Gassendi too: "for the Stars to appear to travel westward, it matters not if they do move that way in reality $[$ reipsa] or it is the spectator's eye that travels eastward with the Earth. The Stars will appear to move west in either case" $(1675,135)$.

Completism was also the view of most theorists of mechanics then. Opponents and defenders of the new 'mechanical philosophy' granted it alike. "To move and to not move are contradictory predicates, which is plain. So, by [my] Axiom 1, they cannot both be in [a subject] at the same time," argues Honoré Fabri, an eclectic $(1646,12)$. Wren assumes Completism as he sets out to derive the laws of impact. When two bodies collide, their "Velocities proper and most Natural are inversely proportional to the Bodies," he asserts (Wren 1668, 867). Mariotte too, in 1679: "The relative speed of two bodies is that whereby they approach or recede from one another, no matter what their proper velocities may be" (1740, 3). Newton endorsed Completism even before the Principia: “[A body's] centrifugal endeavor is always certain and determinate; which proves [arguit] that there is some certain and determinate quantity of real motion in single bodies [motus realis in singulis corporibus], a motion wholly independent from relations to other bodies, which are as innumerable as the relative motions they constitute" (1684-85, 25r). Huygens in 1694 tells Leibniz, "I saw you believe that 'it is absurd to think there is no real motion but only relative." " Leibniz agreed: "motion, or rather the moving force of bodies . . . must 
have a subject" (Huygens 1888-1950, 10:606, 639). Leibniz then admitted to Clarke, "I grant there is a difference between an absolute true motion of a body, and a mere relative change of its situation with respect to another body" (Alexander 1970, 74). So, bodies have unique true, or real, or 'absolute', motions whereby they either move truly or rest but not both.

Endorsing Completism comes with the attendant duty to explain the nature of true motion. In response, some reached for Absolutism, claiming that true motion consists in motion in Absolute Space. Borelli preceded Newton by 2 decades, in a tract on collision mechanics: "Local motion occurs either from one place of world space to another; or in the relative space of some container. The former shall be called real and physical motion [motus realis \& physicus], and the latter relative motion [motus relativus], though often it does not involve a change of position in the place, or the space, of the world" (Borelli 1667, 2). Newton too argued, "true motion does not consist at all [minime consistit] in relations to other bodies." So, it "cannot be defined [definiri] except in terms of immobile places," the parts of Absolute Space (Newton 1687, 8). Euler then rediscovered independently part of Newton's case for Absolutism and concluded, "If a body occupies successively one part of this immense space after another, it moves; but if it persists continually in the same place, it rests" $(1736,2)$.

T-Relationism was famously Descartes's doctrine. Any body has a motion or rest "in the proper sense," he asserted, "in accordance with the truth of the matter [ex rei veritate]." Allegedly, it consists in the body's transference "from the vicinity of the bodies that touch it immediately, and are regarded as at rest, to the vicinity of other bodies" (Descartes 1644, 46). A century later, Emilie du Châtelet likewise espoused T-Relationism, in a peculiar version. "Absolute motion is a Body's successive relation to other Bodies, regarded as immobile, and that is its real motion properly so-called" $(1740,215)$.

Relativism was then by far the least popular position. The young Leibniz asserted it: "Concerning motion I have shown elsewhere that it is not possible to determine which subject it is in," he wrote circa 1678 in a note (2001, 257). But, he kept it private, and once he discovered "force," he seems to have abandoned it in favor of T-Relationism.

4. Huygens on Relative Motion. Huygens in Codex $7 A$ insists at length that all motion is relative. With the four views above as backdrop, we must ask, what is the real content of his claim? Did he endorse T-Relationism or Relativism? Recall that they are logical contraries, so it is crucial to establish which of these two his considered view was.

Huygens denied Absolutism vehemently, which has led many to think his target was Newton's absolute space. But, that misses the point: what Huygens rejected is really Completism. "We cannot conceive in any way what the true and simple motion of a single entire body is - nor does it differ 
from the state of rest of the same body" (9.24r). And also, "Mariotte distinguishes the relative celerity of two bodies from their 'proper' celerities. I claim there is no proper celerity" (10.a). By turns, he asserts that true motion is nonexistent, empirically inaccessible, and superfluous for mechanics:

Indeed, if we look carefully into the nature of motion, we shall find that this true motion and rest, as nearly all of them understand it, not only cannot be known, but does not exist at all in the universe. (7)

[We] cannot ascertain at all the quantity of that true motion they imagine for themselves. This quantity, in fact, they cannot state. (1.b)

In vain does one ask what this true motion is - for what good is it? It is the usefulness and consequences of our principle - namely, that motion is only relative - that are the marks of its worth. (6.i)

In sum, Huygens denied true motion, so his view was Relativism- the thesis that motion has no subject, so it is an irreducible relation, not a property of single bodies, and that bodies have no privileged velocities. Ergo, his real target was Completism. A passage in a late letter to Leibniz confirms it. Huygens there says that Leibniz believes, "when several bodies move relative to each other, each has a certain true degree of motion or force, in which I disagree with you" (1888-1950, 10:646).

Granted, this looks unimpressive. All we have seen from Huygens so far is blanket denials and irked denunciation. The real philosophical value of his notes, I submit, is in their ability to cope with the two hardest problems for Relativism: giving an account of inertial frames and explaining how rotation is relative motion. It is to these that I turn next, to show that Huygens succeeds.

Before I do so, I introduce some definitions, to make his thought fully clear. A-MOTION: actual change of relative distance over time between two or more particles. L-MOTION: actual rest between rigidly connected particles but actual motion if the constraints were removed. Its correlate is L-REST: actual rest over time between the parts of a rigidly constrained system, if the constraints were removed. ('A' stands for 'actual' and ' $L$ ' for 'latent'.) H-FRAME: a set of four noncoplanar particles kinematically unconstrained, mutually at rest over time, and unaccelerated. C-FRAME: a rigid body or set of particles with rigid constraints. ABSOLuTE SPACE: an enduring rigid, immobile, infinite frame ontologically distinct from matter. I take their geometry to be Euclidean.

5. Inertial Structure. A body counts as accelerated in manifold countless ways, depending on the standard to which we refer its motion. However, in classical theory only some accelerations are objective, that is, correlate invariably with mechanical verae causae as their kinematic effects. Specifically, objective accelerations are changes - in speed, direction, or bothfrom inertial trajectories. Hence, to secure objectivity for his mechanics 
Huygens must explain which relative motions count as inertial paths, so that objective accelerations can be defined from them, and so begin to point us to their underlying causes.

Not until the late 1680s did Huygens feel the force of this question. When he finally does, he puts it as the task of explaining what it is for bodies to move in a straight line - and in different directions. ${ }^{4}$ That explanation, in turn, would allow him to give objective empirical meaning to forced motion-specifically, change of speed of rectilinear translation or deflection from inertial paths. To that end, Huygens introduces a technical notion, namely, "bodies at mutual rest" (corpora inter se quiescentia). I explicate it next, and then I examine its epistemology.

Constitution. "Bodies at mutual rest" are any two or more solids satisfying two conditions. First, the bodies must be kinematically free. Huygens means it as a blanket imposition, unrestricted to specific physical constraints like rigid rods, flexible lines, or immobile surfaces. His bodies must be "not tied together," must be situated "where nothing can impede their motion" and be "entirely free to move," namely, move "separately and in no way bound or joined together" or "kept together by any bond or obstacle" (6.a, 12.33r, 11.d, 7.19r). They must be generally unconstrained.

Second, they must "keep the same situation and distance relative to each other" over time (7.19r). This is really two conditions. The mass centers of the constituent bodies must keep their relative distances. And, the masses themselves must not spin around internal axes. In Huygens's words, "those bodies are at rest relative to each other that, not being held together by any bond or fastening bar, keep the same relative distance, both as a whole and with their parts" (8.b).

Do these masses accelerate? If they do, then Huygens is mistaken in resting the Law of Inertia on them. Four arguments collectively imply that "bodies at mutual rest" are nonaccelerating. Before we see why, keep in mind that only contact action - the "true and sound Philosophy," as he called it - is legitimate in Huygens's mechanics: from collision and vortex pressure. One argument is from silence: in general, Huygens says so if he takes bodies to be colliding. ${ }^{5}$ But, he never says of "bodies at mutual rest"

4. Nowadays arc-length straight lines are said to be the paths of force-free bodies. But, Huygens's mechanics is not a theory of (Newtonian) impressed forces. So, I use the phrase 'force-free' to denote bodies that are noncolliding or on which ether pressure is balanced by some mechanical agency.

5. For example, "If we make two bodies collide that were previously moving, together with us, with a common uniform motion, after the impact these two bodies will rebound or move together relative to us (who are being carried with the same common motion) in no way differently than if that motion adventitious to all were lacking entirely" (7.15r). 
that they collide; hence, they do not accelerate by that mechanism. Another argument is that Huygens meant these body sets to underwrite the various 'lab frames' of his impact mechanics: the river bank, the boat floating on it, as experiments with colliding bodies unfold inside, and so on. But, he takes his lab frames to be at rest or in uniform translation relative to one another, never accelerated. ${ }^{6}$ Yet another argument is from Charity: were "bodies at mutual rest" accelerated, Huygens's basic dynamical laws would be evidently false, and he is too perceptive not to have seen that. ${ }^{7}$ So, he took them to be nonaccelerated.

In fact, "bodies at mutual rest" are unaccelerated. The only sources of momentum transfer in Huygens's mechanics are collisions and ether pressure. Ether vortices induce central acceleration fields, which would disturb the mutual rest of free bodies, thus violating the defining conditionalthough vortices themselves can be relatively stationary and will remain so. ${ }^{8}$ Objection: bodies remain at mutual rest even as they receive equal and parallel accelerations. Answer: that scenario is disallowed by Huygens's impact mechanics. ${ }^{9}$ An accelerated stream dragging along - by collision-a set $G$ of stationary bodies will disturb their mutual rest, save in two cases, and both can be ruled out. One is to require that $G$ 's members have equal masses, congruent shapes, and parallel placements in the stream, but of course Huygens does not allow such arbitrary restrictions. His account is fully general, not meant to depend on good luck. The other is a physical impossi-

6. For example, "If I inquire if, on a boat moving uniformly, the impact and rebound of bodies will occur in the same way as on a boat at rest- to be sure, in each case I understand 'to move' and 'to be at rest' relative to the earth and the riverbank" (1.b). 7. Relative to a set $H$ of (smoothly) accelerating 'bodies at mutual rest', a force-free test body will appear in free fall or projectile motion, depending on the accelerations on $H$. These paths are just what Galileo and Torricelli had treated, in 1638 and 1644, respectively. Huygens was by far the subtlest reader of Galileo and Torricelli. Charity dictates that we give him this: if $H$ accelerates, force-free bodies trivially fail the Law of Inertia relative to it.

8. In modern terms, a Descartes-Huygens vortex is a 'hard shell' attractive potential. At cosmic scales, vortex centers are outside each other's 'shell', so they remain at mutual rest.

9. Although it is permitted in Newton's theory, with action-at-a-distance gravity from a field source at infinity-hence, the need for Newton's Corollary Six to his laws of motion. The 'mechanical' model of terrestrial gravity in Huygens's 1690 Discours de la cause de la pesanteur induces nearly parallel accelerations: the angles they make with true parallels are negligible in practice, although not in theory. I leave out here whether his notion can cope with universal gravitation. In the spirit of Contextualism, I restrict my account to Huygens, who thought there was evidence against universal gravity (Schliesser and Smith, forthcoming). 
bility: having single jets in the stream self-adjust their momentum to the mass of the particular $G$-member they carry along, or else relative accelerations arise to disturb mutual rest. ${ }^{10}$

And, they are nonrotating too, not just unaccelerated. For Huygens, it is constitutive of rotation that particles "endeavor to recede from one another, and strive to do so with all the more force as their relative motion is greater" (app. II.173v). In a set of unconstrained bodies, rotation would cause relative drift. The defining condition thus ensures that "bodies at mutual rest" do not rotate.

And so, Huygens has taught how to set up an inertial frame, in which an observer at rest sees all force-free bodies remain in uniform translation. That was his intention all along:

If a body moves freely and without obstacle in respect to those bodies at rest relative to each other, the moving body will run over a straight line relative to them, and will be translated by a uniform motion. ... From this motion, referred to bodies at rest relative to each other, one can at last understand and define [definiri] what it is to move freely [libere] and uniformly [aequabiliter] in a straight line. ... Assuming some bodies to be at rest in regard to each other, many other bodies can move relative to them along various straight lines, and so these lines will be called the various directions of motion. (7.19r)

Relative to [free bodies at mutual rest], bodies in motion describe a straight line, move uniformly and persevere in this state. This has the status of a Principle. From it is to be understood what it is to move in a straight line. On different directions. (8.c)

Because 'bodies at mutual rest' are neither accelerated (in ways allowed by his mechanics) nor rotating, it follows that for Huygens the Law of Inertia is true relative to a preferred set of frames. Thus, his view is importantly distinct from two modern conceptions: one that takes the Law to postulate the existence of an inertial frame and one that regards it as defining the frames suitable for mechanics.

Now I must make a friendly correction. His frames lack sufficient kinematic structure: they cannot identify motions univocally. The number of "bodies at mutual rest" required for that task is four. But, Huygens allows frames designated by just two or three bodies. That is enough for his immediate aim - a mechanics of direct, particle collision - although not for a

10. By the Second Law, an accelerated stream of equal-momentum jets induce in unequal test bodies tidal forces, i.e., unequal accelerations, which disturb mutual rest. To avoid this consequence, each jet must self-adjust its momentum to the individual mass it strikes. I thank Katherine Brading for pressing me on this point. 
general theory of extended bodies. Fortunately, we can redress his shortcoming without much anachronism. Just decades later, Euler showed that, to determine all positions - hence all possible motions - univocally, we need no less than four noncoplanar points. So, he inferred, "if we know the distance of a point $O$ to four other points $A, B, C, D$ not all in the same plane, its position is determined completely" (Euler 1765, 4). Hereafter, I take "bodies at mutual rest" to denote a set of four nonspinning solids. Then they count as an $\mathrm{H}$-frame as I defined it, so I will call them by that name.

Huygens now makes a crucial point. His $\mathrm{H}$-frames may be at rest or translating uniformly relative to one another, but none is 'truly' at rest or has a true velocity. We may take any such frame as stationary, arbitrarily: there is no fact to confirm or disprove our choice. For instance, suppose the stars are an H-frame; "there is no reason why we should not say that the fixed Stars are at rest among themselves, relative to each other. But then, apart from this rest, we cannot ascribe to them any other rest one ought to call true. The same argument applies to any other bodies, which obviously can be said to be at rest only in respect to one or several other bodies - relative to which they keep the same position and distance" (7.17r). Likewise, suppose - as Huygens, following Kepler, once thought - that a comet translates uniformly in respect to the stars. "How are the stars any more at rest than some comet traversing the skies in a straight line (as Kepler shows)? For the latter could be considered stationary just as much as the entire host of the stars" (3.a). Thus, there is no distinguished standard of rest; all $\mathrm{H}$-frames are dynamically equivalent.

An evaluative point is in order here. Note that $\mathrm{H}$-frames are compatible with Newton's Corollary Five. Suppose that $J$ and $K$ are two H-frames in uniform translation relative to one another. Then every constituent particle in $J$ changes position toward $K$ by the same amount in a given time. Hence, the particles stay at rest inter se, so $J$ remains an $\mathrm{H}$-frame. Mutatis mutandis for $K$ in respect to $J$. Ergo, H-frames in relative uniform translation obey Corollary Five: "A space being given, the motions of bodies in it are the same among themselves [inter se], whether the space rests or moves uniformly ahead without rotation" (Newton 1687, 19). More exactly, let two systems $\mathbf{S}$ and $\mathbf{T}$ be individually closed and mechanically identical, that is, have the same reference configuration and distribution of mass, internal forces, torques, and stresses. Let $\mathbf{S}$ have the same placement and initial velocities relative to $J$ as $\mathbf{T}$ has relative to $K$. Then Newton's laws entail that, for all subsequent times, the placement and configuration of $\mathbf{S}$ in $J$ will be the same as that of $\mathbf{T}$ in $K$. And so, in effect Huygens could show that his $\mathrm{H}$-frames can secure as much inertial structure as Newton's dynamics really needs. From the dynamical laws in the Principia, Huygens's 'bodies at mutual rest' entail the same empirical content as Absolute Space. (They are inertial frames, after all.) And, Huygens can do that without Newton's two 
most contentious notions: true velocity, an empirically inaccessible quantity, and Absolute Space itself, a philosophically divisive entity.

However, Huygens has no account of an inertial clock-no one then who opposed absolute time had one. That makes his account of inertial frames incomplete: he cannot give empirical meaning to the notion of crossing equal distances in equal times. Set aside this (admittedly important) lacuna for a moment, and let us sum up the source of kinematic-inertial structure in his highly nontrivial mechanics: it is the set of all H-frames, which are Galilean and form an equivalence class. That supplants true velocity and Absolute Space, he thinks.

Epistemology. So far Huygens has answered a conceptual question: What is an inertial frame? This problem has an empirical correlate: Do any exist? He realizes that maybe none exists after all: "Any free body is either at rest or moves uniformly in respect to other bodies themselves at rest relative to each other, if they exist - or it would move so, if they existed" (8.b). Now, suppose that an H-frame does exist. How might we establish that? In Huygens's mechanics, a conclusive answer would be to find four nonconstrained solids at mutual rest over time. Short of that, we must content ourselves with considerations of greater or lesser plausibility. With that in mind, Huygens thinks there is an excellent approximation of an H-frame: "we know that the fixed stars are mutually at rest and have not received any impulse to move round; for in that case they would recede from one another." But, the stars would recede "unless they are all embedded in a solid sphere, as some used to believe in the past" (11.g). It was a highly implausible supposition at the time (refuted in 1718 with Halley's discovery that stars have proper motions). Still, if true, it would disqualify the stars as a suitable frame, as Huygens freely admits. In conclusion, inertial frames exist to the extent that his constitutive conditions - kinematic freedom and mutual rest - obtain empirically and insofar as we may extend his doctrine to include the notion of an inertial clock.

6. Rotation. Given that he thinks there are no preferred velocities, Huygens must explain how purely relative motion can cause dynamically distinguished centrifugal effects. He thinks he can: "Now I move on to circular motion, to show that what they call true motion is not found here anymore than in rectilinear motion but it too is relative" (11.d). Huygens responds in two parts. He argues that rotation is relational, not a property, and is no true motion; that is, single rotating bodies have no unique, preferred total velocity. In effect, he aims to show that rotation is relativistic, so as to defeat Completism at last.

He fulfills his task with mixed success. That is because a sharp look at his results reveals not one but two accounts of what rotation is, and they differ 
in crucial respects. In the first, an $\mathrm{H}$-frame is indispensably in the picturethe motion is described relative to it. The second account lacks an H-frame, and the motion is referred to the very parts of the rotating system; to an inertial observer, the direction of this motion is radial.

The First Account. The fragmentary state of Huygens's notes makes it hard to tell what his official view of rotation was. The more frequently stated - the soundest, too, and recognizably modern - is this analysis:

Circular motion is a relative motion along parallel straight lines, with the direction changing continually while the distance remains the same because of the bond. Circular motion in one body is the relative motion of its parts while the distance remains the same because of the bond. (6.d)

Circular motion is that which takes place in respect to a point taken to be at rest, while the mobile's distance to it remains the same because of the bond or obstacle, but with the direction changing constantly. The celerity of a body (or of some part of it) in respect to that point at rest is measured from the bodies at rest relative to one another and to that point, taking into account the time and the length of the circular path traversed [amplitudo circuitus]. (8.a)

And we can define the number and celerity of rotations in regard to other bodies at rest relative to each other. (7.19r)

Huygens's epitome of rotation is spin, not orbital motion. He explains it with two bodies rigidly connected revolving in a plane around their common mass center or a body spinning around an internal axis. For such settings, I construe his words above as follows. A body rotates just in case its parts rotate relative to an $\mathrm{H}$-frame or set of 'bodies at mutual rest'. Really, they rotate in respect to an instantaneous axis at rest in an H-frame. And, every part has a conatus, or endeavor to continue in a straight line defined relative to the frame. That conatus has a direction and a size. ${ }^{11}$ Its direction is tangential to the particle's trajectory in the H-frame. That is clear by the terms of the account so far and from figure 1, an improved rendition of Huygens's own drawing. Its size, or scalar value $v$, is proportional to the 'centrifugal force' $\mathbf{V}$, the particle's endeavor to recede radially from the center

11. The direction is the line of its instantaneous endeavor: "This line along which a body (or any of its parts) moves - or would move if it were free - we call the direction of motion" (8.20r). Huygens knows that some dynamically relevant quantities of motion are vectors, not scalars. For example, he grasps linear momentum clearly: "our principle could be as follows: that as much motion must remain [throughout the collision]. But it must be understood in the sense that as much motion remains in the same direction" $(12.33 r)$. 


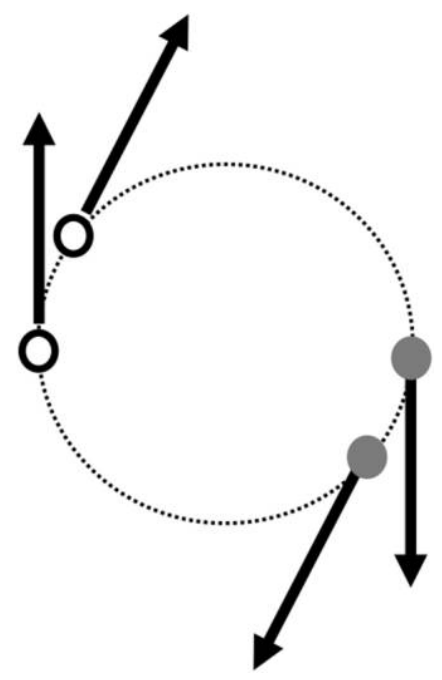

Figure 1. Rotation as 'change of direction'. Two particles (one gray, one white) are in rotation. Each is shown at two consecutive positions. The arrows are their tangential velocity vectors.

of rotation $C$. Huygens in De vi centrifuga had proved that, for a unit-mass particle,

$$
|\mathbf{V}|=\frac{v^{2}}{r}
$$

where $r$ is scalar distance to $C$ assumed stationary in some H-frame (1728, 114).

What makes it motion? Huygens really answers this twice. First, he claims it is a 'constant change in direction'. He means change in the direction of conatus, the tangential velocity vector. For any two diametrically opposed particles $A$ and $B$ in a rotating system, their conatuses are parallel and contrary (again, see fig. 1). Over time, they remain so, but the direction in which each particle points individually changes at every instant; the spin being uniform, the change is constant. Huygens calls it the 'direction changing constantly'. Second, Huygens claims the particle moves in that it changes position relative to the frame. In that sense, its motion is an arc-length displacement. Thereby, his account grounds - rather incompletely, absent a notion of inertial clocks - two important modern measures of (uniform) rotation: angular speed and centripetal acceleration. In his words, they are amplitudo circuitus and change of direction 'in the first instant'.

The Second Account. Although embryonic, the analysis above is sound and coheres with his notion of inertial frames. And yet, Huygens ended up 
of two minds about rotation; a hard look at Codex $7 \mathrm{~A}$ reveals another account. To soften up the reader, I begin with these declarations:

The relative celerity of bodies measured along lines of direction is one thing, whereas celerity measured by the change in distance between the bodies themselves [inter se] is another. (8.a)

But can two bodies, whose mutual distance stays the same, move relative to each other? Certainly, when the increase in their distance is impeded. (9.24r)

Maintaining a definite position among themselves is not a mark that bodies are at rest relative to each other. (11.c)

For if bodies are linked or held together, they can maintain their reciprocal situation and yet be in motion relative to one another. . . Thus, [two constrained rotating bodies] A and B will have a motion between them, i.e. relative to each other, but without a change in position or distance between them. (11.d-f)

I submit that Huygens means 'relative motion' here in a new sense, namely, as L-motion as I defined in section 4. Two rigidly constrained particles move relative to each other because they would increase their relative distance actually, provided the constraint was dissolved. Huygens's justification for his unorthodox usage is that this kind of latent relative motion comes from actual kinematic change - which becomes constrained - and it would turn back into actual change, upon removing the constraint. To that end, he offers a thought experiment (see fig. $2 b$ ). Let two particles in uniform translationdefined relative to an external $\mathrm{H}$-frame - move in parallel but opposite directions. Let an inextensible string (filum) with hooks at both ends lie in their path normal to both trajectories. Let the particles collide with the string, and so become hooked, at the same time. Thereby "the bodies' rectilinear motion turns into circular motion, which the string . . . will reveal by the tension in it" (6.j). Before their capture, the particles have a mutual speed, or rate of actual change in mutual distance; this mutual change is defined in terms of each other, not relative to the $\mathrm{H}$-frame. The closer they get to the hooked line, the closer that speed gets to zero. And yet, they are still in motion relative to each other: "the distance between them changes only a very little - virtually not at all. The same thing happens in circular motion" (9.b). Introducing the constraint does not destroy or annihilate that mutual relation. Rather, it just suppresses it, or makes it latent. As proof, just cut the string, thus restoring the particles' kinematic freedom: their actual change of mutual distance will resume. This shows that (latent relative) L-motion is well defined: suppose I come across two tethered bodies and cut the string between them, and yet they keep their mutual distance. These bodies then count as having been at L-rest before I removed the constraint. L-motion thus has an empirically meaningful correlate, so it is itself meaningful. 

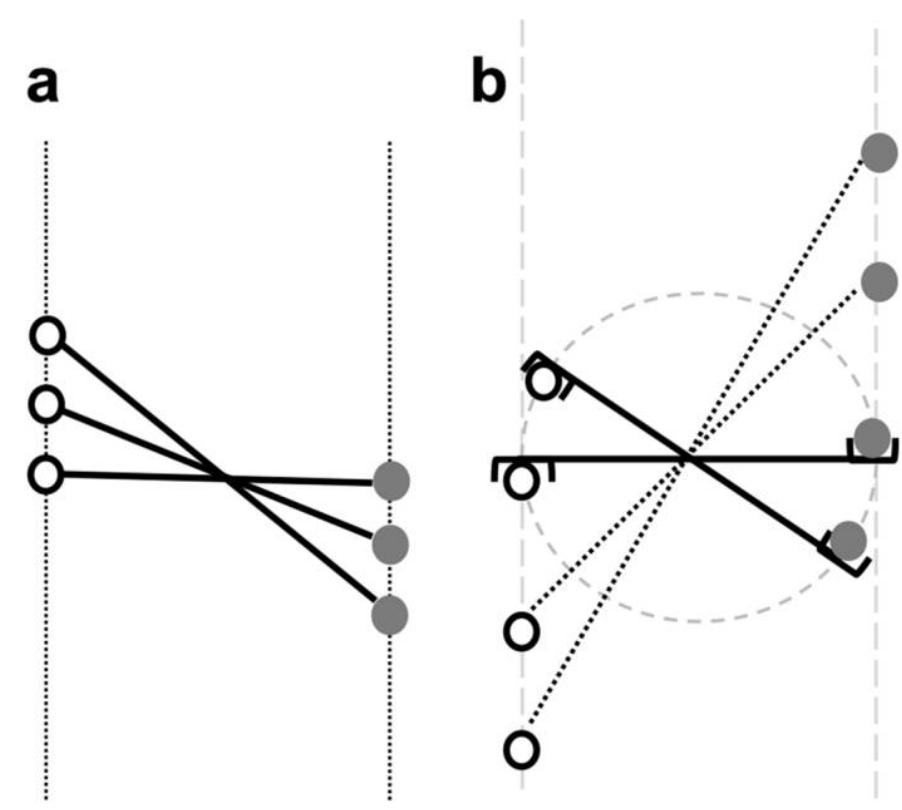

Figure 2. $a$, Rotation as L-motion, relative to each other. Suppose they were constrained by a rigid rod, which is then removed. The solid line is their relative distance postremoval; it measures the 'relative celerity' as Huygens conceives it. The dotted lines are their individual trajectories as seen from an inertial frame. $b$, Rotation as relative motion. This is Huygens's illustration of how rigid mutual rest ensues by suppressing actual mutual-relative motion and would turn back into it if we removed the rigid constraint. So, he infers, rigid rest can be relative L-motion.

This L-motion is radial: if a rotating particle's 'endeavor to recede' is analyzed as L-motion, its direction is on the line between the corotating particles. It is the line of 'mutual speed' as Huygens defines it above and also the line of the dynamical effects induced by this relative motion, for example, tension, if we replaced the rigid constraint with a spring or a strain gauge. ${ }^{12}$ But clearly, to all inertial observers the line is a diameter of the spinning system.

Although he never mentions Newton in this context, I think it is very likely that Huygens devised this scenario in reply to Newton's Gedanken-

12. Recall that for Huygens 'centrifugal force' is that which is balanced by the tension in the cord keeping a body in orbit around a center. The tension is along the cord and so is the 'centrifugal force' of rotation. He conceives 'centrifugal force' as an endeavor intrinsic in the body, which has it just because it moves - not because an external agency caused it to have. That 'force' drives a rotating body to move on the involute of the (circular) path it has when seen from an inertial frame. As Stein puts it beautifully, the involute "leaves the [path] at right angles" $(1977,7)$. But, that endeavor to move so appears only to observers in a C-frame that rotates with the body. Inertial observers will see the body strive to move along the local tangent to the trajectory, not normal to it. 
experiment of the two globes spinning in a vacuum. Huygens's aim was to generate an intuition: relative motion can be L-motion too, not just A-motion. He needed it for his second account of rotation, which does not rely on H-frames. Although less represented in Codex $7 A$ than the first account, it is still there. Consider these passages:

Circular motion is the relative motion of the parts, hurled in opposite directions but held together because of the bond, or connection. (9.24r)

Yet the parts of a body can move relative to each other while the distance between them remains the same, on account of some bond or barrier; this is called rotation [motus vertiginis]. . . . In this motion, the parts endeavor to recede from one another or from a point defined with respect to them; and they strive to do so with all the more force as their relative motion is greater. Likewise, it is from this force that we can judge the quantity of this relative motion, seeing as we cannot do so from the change of distance. (App. II.173v)

The view they entail is this. Rotation is a mutual relation between corotating bodies or particles. They rotate just in case they tend to recede from each other. And, they just tend to recede - the constraint prevents them from doing so in fact.

Then what makes rotation in this sense a type of motion? After all, the particle is at rest relative to its corotating siblings, because of the constraint. I take Huygens to claim that rotation so analyzed is relative L-motion, that is, just counterfactual change: although actually at rest, the particles would increase their mutual distance upon removing the constraint, so they are in latent motion.

As a species of motion, for every particle this L-motion has an instantaneous size and direction. The size is proportional to its 'centrifugal force'. The direction is that in which they would move if the constraint was dissolved. In a two-particle system, the direction is on the line between them. That line is the only empirically meaningful direction in this account, because $\mathrm{H}$-frames are absent by definition: "in the absence of those bodies [at mutual rest], from centrifugal force we know that the greater this relative celerity, the greater the centrifugal force. From it, we can discover circular motion," Huygens claims (8.a). See also figure $2 a$, based on Huygens's sketch, showing two particles at three consecutive times, with the constraint dissolved. Their relative motion is the increase in mutual distance (i.e., the solid line between them). Again, the increase is counterfactual-actual mutual rest but latent change of mutual distance. Only by removing constraints would that latent motion become actual relative change.

Note a key fact: Huygens's second account is in a rotating frame. Let an observer $O$ rest on the 'line of mutual speed' between the particles (i.e., the 
line of their L-motion). To all inertial observers, $O$ herself counts as rotating; thus, $O$ and the rigidly connected particles make up a rotating $\mathrm{C}$-frame. In this description, the particles' centrifugal force too is radial-it makes them tend to recede along the radius from the center of spin. (In contrast, to inertial observers the particles' endeavor is along their respective tangents, not the radius.) Ergo, for this analysis of rotation, the relevant frame is noninertial: an unconstrained force-free body in it will describe a curve, not a straight line, as Huygens knows too well. See figure 3, based on his De vi centrifuga, showing the trajectory of a force-free body as seen by an observer in a rotating $\mathrm{C}$-frame. As Huygens proved, the path is a cycloid.

Call the first construal above 'A', the second ' $L$ '. The two accounts are wholly distinct; to see that, juxtapose them in a nutshell:

A-ROTATION: a type of A-motion relative to a nonrotating H-frame. Actual kinematic change in respect to an inertial frame. Its objective direction is tangential to the trajectory.

L-ROTATION: a type of L-motion relative to a rotating C-frame. Nonactual, latent kinematic change in respect to a noninertial frame. Its objective direction is radial.

And yet, Huygens asserts both nearly in one breath. His keen, exquisite physical insight makes it incredible that he missed their drastic differences.

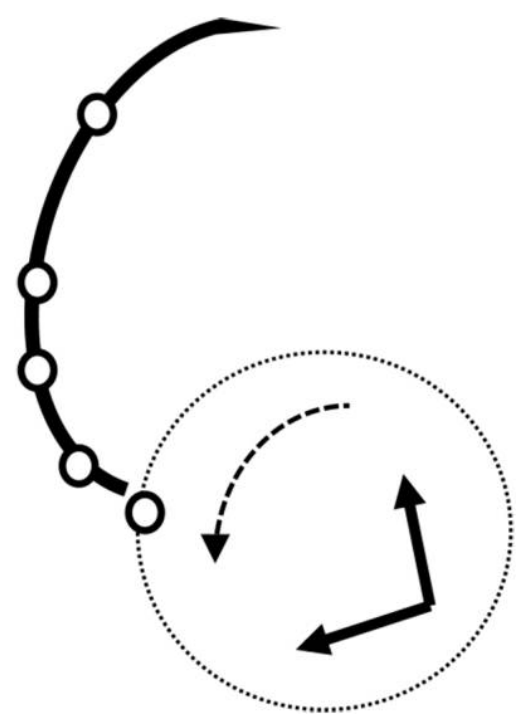

Figure 3. Path of a zero-force body as seen from a rotating frame (uniformly counterclockwise). 
That he restated both in his notes attests to how exerted he was by Newton's two globes rotating in empty space. ${ }^{13}$

The other half of his case that rotation is relativistic argues that it is not true motion. To assess it properly, we must know its real target. Huygens subverts a subthesis of Completism, that is, that true motion is the "whole motion" of a body: its total velocity at some given instant and effective trajectory over time. Newton had made that premise explicit in his third 'argument from the properties' of true motion. We must posit immobile places, he had argued, to vindicate the notion that bodies have "whole and absolute motions [motus integri et absoluti]," namely, true total velocities (Newton 1687, 8). Huygens denies that bodies have 'whole motions'. The apparent motion being given, his theorems on centrifugal force let us compute a rotating body's velocity (linear or angular) relative to an axis $C$ assumed stationary in an $\mathrm{H}$-frame. Now, this frame-hence a body rotating in it - could well have linear velocities toward other H-frames. Those must be vector added to the body's rotation to compute its 'whole motion'. But, by Newton's Corollary V, no mechanical experiment can detect 'true' linear velocities of one H-frame relative to another. So, the 'whole motion' is incomputable, having empirically inaccessible parts. "For even they grant that a spinning body, be it an entire body or one made up of conjoined parts, can at the same time move rectilinearly with a motion that carries all its parts. But they confess that there is no mark whereby we can discern to what extent this rectilinear motion is true motion," objects Huygens. Then rotation is not true motion in that sense. It is relativistic: "Without being able to say how much either [corotating] body has of this motion commonly called true [véritable], and without having this sort of motion at all, for it is nothing but a chimera grounded in a false idea" (6.d, 11.g).

My construal allows me now to solve a famous difficult puzzle about Huygens. He says, "I had long believed that the kriterion of true motion lies in circular motion. How I came to change my mind" (5.e). The puzzle is, exactly in what respect did he change his mind? I propose an explanation. The young Huygens used to think that rotation is evidence for Completism, in that it is a fact about single bodies in true (circular) motion. However, Huygens then had no view about the nature of true rotation: he endorsed neither Absolutism nor T-Relationism. Some time between 1686 and 1691, he found a way to analyze rotation as an irreducible relation-be it to an

13. A-rotation and L-rotation are logically compatible, but the latter is dynamically inadequate. The L-motion of two rotating particles is accelerated - remove the constraint, and their mutual distance changes nonuniformly, which Huygens knows: "as they recede [from each other], their [mutual] celerity increases continuously" (7.17r). But there is no real cause for their mutual acceleration. So, it counts as inertial (L-)motion, and yet it is not uniform. 
H-frame or to a corotating body_ and so he realized it does not entail Completism after all. Rather, it supports Relativism. ${ }^{14}$

7. Hermeneutics. Ceteris paribus, my interpretation is preferable to Stein's: I have explained Huygens without anachronism, fully and in detail, and with no need to ignore or dismiss his explicit assertions. Moreover, Stein and I differ radically on the nature of rotation: his Huygens conceived it - ineffably, we must believe - as a difference in the four-velocities of rotating particles. I have shown from direct evidence that Huygens had two accounts. Using different methods, we obtained distinct construals of his concept 'relative motion'. I explained it as A-motion and L-motion, in terms available to Huygens; Stein, as a difference of space-time vectors, in terms that Huygens could not have had. I agree with Stein that, among the early moderns, Huygens rivals Newton in depth of insight into the foundations of classical particle mechanics. But, I went beyond him in explaining the material resources Huygens uses to anchor that structure, that is, the notion of 'bodies at mutual rest' and his two kinds of relative motion.

Critically, my reading allows me to absolve Huygens of two grave accusations and to subvert two myths about him. The first charge comes from Earman, who complained that Huygens "flatly contradicts himself." Allegedly, he did not refer rotation "to some reference body" and yet admitted that rigid spin is eo ipso mutual rest, not relative motion, Earman claims (1989, 71). My evidence shows that his two claims are false, and so the charge is untenable.

Barbour accused that there is a "serious contradiction at the heart of Huygens's doctrine," which "seems to have escaped him completely." Supposedly, Huygens's claim that motion is relative-which Barbour reads as, all kinematic quantities are relativistic - contradicts his recognition that some motions (i.e., inertial translations) are privileged (2001, 672-73). As evidence, Barbour pointed to Huygens saying, "a body's motion could be truly equable and, at the same time, truly accelerated, according as we refer it to different other bodies" (1888-1950, 6:327). Unfortunately, Barbour misread Huygens's words. The real context of his claim is rotation. Consider the white particle in figures 1 and 2. Analyzed as A-rotation (relative to an external H-frame), its motion is uniform; analyzed as L-rotation (relative to its corotating gray particle), its motion is accelerated, as I explained at the end of section 6. So, Huygens implies, the same phenomenon-spin-is both uniform and accelerated motion. Still, that is because Huygens has described the same process in two kinds of frames. But, the Law of Inertia fails in

14. An alternative explanation: Huygens used to think rotation is motion in Absolute Space but later came to see that it is relative to matter (Stein 1977, 10). On the basis of my evidence above, this must be false. Huygens never accepted Absolutism. 
the second kind. Then if we restrict ourselves to his preferred account (i.e., A-motion relative to some $\mathrm{H}$-frame), linear acceleration is objective and invariant, not relativistic — and so is inertial motion. So, Huygens is innocent of Barbour's charge. ${ }^{15}$

The first myth, from Lange in the nineteenth century to Chareix in the twenty-first, is that Huygens's key problem was "is motion absolute or relative?" (Barbour 2001, 478). Now it is clear that they are mistaken. His real question was whether bodies have true motions (i.e., preferred velocities) a view he subverted at length: "it seems greatly important to try and root out this common error," he proclaimed (7.15v).

A second myth: the Principia was the chief impetus behind Huygens's case for Relativism, which he allegedly articulated to answer Newton, with Leibniz as a third interlocutor. My interpretation shows that this, too, is false. Huygens had encountered Absolutism and rejected it out of hand already in the 1660 s, well before the Principia. In those years William Neile had pressed on him several arguments for the existence of motion in $\mathrm{Ab}$ solute Space, which Huygens in Codex $7 A$ keeps refuting. ${ }^{16}$ And, he had also come across Absolutism in Borelli's De vi percussionis of 1667. To Huygens, Newton's case in the Scholium appeared as a late restatement of an old view - a mistaken one, he thought — updated just by the addition of the globes scenario. Huygens's real target was not Absolute Space but Completism, and he rebuffed all natural philosophy-Aristotelian and early modern, astronomy and dynamics - for its presumption that bodies have true motions. From that angle, Newton (who assumed it) was just one among many, and Huygens mentions him as an afterthought. "It is commonly believed that there is some true motion, as opposed to relative. Borelli, Mariotte. Maybe Pardies too? Newton thinks so. Wallis, perhaps? .. . Thus believe all of them; and also Newton," Huygens asserts (v.E, x.28r). This myth about Huygens is as false as the first, then. ${ }^{17}$

Finally, a corollary of my interpretation is that Earman's statement of relationism is insufficient in two respects. First, it cannot distinguish TRelationism from Relativism, two distinct theses, logically and historically. R1 fails to make conceptual room for the (early modern) view that some relative motions are preferred: many canonical figures denied absolute space and yet asserted that there is a fact about whether the earth, the sun, and the

15. Recall as well his charge that Huygens failed to explain relative to what a force-free body moves in straight lines. This too is unfounded. As I explained, they are straight relative to some arbitrary $\mathrm{H}$-frame.

16. Examples are too many to cite. For context, see Mormino (1993), 54-55.

17. Vilain (1996) and Chareix (2006) also frame Huygens's doctrine in terms of a supposed engagement with Newton, as do nearly all Anglophone commentators. 
stars truly move. ${ }^{18}$ Second, R1 fails to specify whether it means 'motion' as strictly actual kinematic change or it allows latent change as well. As we saw, Huygens uses 'relative motion' to cover latent motions too. R1 should not dismiss that as confused or wrong. For one, L-motion has a well-defined correlate, L-rest. ${ }^{19}$ And physical insight supports it: after all, some motions are free, and some are constrained. For another, Huygens is not alone in thinking that L-motion was legitimately relative motion. Kant too analyzed rigid spin as L-motion of the parts relative to each other (Stan 2015). These facts warrant a reassessment and a more fine-grained reformulation of Earman's R1.

8. Conclusion. I have made a sustained case for Huygens's deep insight into the kinematic content of classical mechanics. Among the early moderns, he saw further than anyone how to articulate mechanical foundations without a concept of true motion. And, he grasped that the phenomena of rotation are not conclusive evidence for absolute space because they can be explained without it. His alternative to Newton is as ingenious as it is defensible. Then we have cause to regret that it remained historically inert. Had Huygens overcome his proverbial reluctance to publish - and had Newton made his De gravitatione public — a profound philosophical exchange would have started 2 centuries before the late modern debates in the twilight of classical physics.

\section{REFERENCES}

Alexander, H. G., ed. 1970. The Leibniz-Clarke Correspondence. New York: Barnes \& Noble. Barbour, J. 2001. The Discovery of Dynamics. Cambridge: Cambridge University Press. Borelli, G. A. 1667. De vi percussionis. Bologna.

Boulliau, I. 1645. Astronomia Philolaica. Paris.

Chareix, F. 2006. La philosophie naturelle de Christiaan Huygens. Paris: Vrin.

Descartes, R. 1644. Principia philosophiae. Amsterdam.

[du Châtelet, E.] 1740. Institutions de physique. Paris.

Earman, J. 1989. World Enough and Space-Time. Cambridge, MA: MIT Press.

Euler, L. 1736. Mechanica. Bk. 1. St. Petersburg.

1765. Theoria motus corporum solidorum seu rigidorum. Rostock.

Fabri, H. 1646. Tractatus physicus de motu locali. Ed. P. Mousnier. Lyon.

Friedman, M. 2013. Kant's Construction of Nature. Cambridge: Cambridge University Press. Garber, D. 1992. Descartes' Metaphysical Physics. Chicago: University of Chicago Press. Gassendi, P. 1675. Institutio astronomica. 5th ed. London.

18. Tellingly, Earman calls Leibniz's version of T-Relationism a "seeming betrayal" of what Earman considers relationism about motion $(1989,43)$.

19. Huygens helps himself to just one species of L-motion, i.e., L-rotation. But there are other species. Consider a nonrotating rigid body in a field of very strong gravity. The tidal forces on the body induce L-translation in its particles. 
Horrocks, J. 1678. “Astronomia Kepleriana, defensa et promota.” In Opera posthuma, 1-239. London.

Huygens, C. 1728. Opera reliqua. Vol. 2. Amsterdam. 1888-1950. Oeuvres complètes. The Hague: Nijhoff.

Lansberg, P. 1651. Commentationes in motum terrae. Middelburg.

Leibniz, G. W. 2001. The Labyrinth of the Continuum. Ed. and trans. R. T. W. Arthur. New Haven, CT: Yale University Press.

Mariotte, E. 1740. "Traité de la percussion ou Choc des Corps" (1679). In Oeuvres de M. Mariotte, vol. 1. The Hague.

Mormino, G. 1993. Penetralia Motus: La fondazione relativistica della meccanica in C. Huygens, con l'edizione del Codex Hugeniorum 7A. Florence: Nuova Italia.

Newton, I. 1684-85. De motu corporum in mediis regulariter cedentibus. MS Add. 3965.5, Cambridge University Library. The Newton Project. http://www.newtonproject.sussex.ac.uk /view/texts/normalized/NATP00091.

1687. Philosophiae naturalis principia mathematica. London.

Riccioli, G. B. 1651. Almagestum novum. Vol. 1, pt. 2. Bologna.

Rynasiewicz, R. 2000. "On the Distinction between Absolute and Relative Motion." Philosophy of Science 67:70-93.

Schliesser, E., and G. E. Smith. Forthcoming. "Huygens's 1688 Report to the Directors of the Dutch East India Company on the Measurement of Longitude at Sea and the Evidence It Offered against Universal Gravity." Archive for History of Exact Sciences.

Stan, M. 2015. "Absolute Space and the Riddle of Rotation: Kant's Response to Newton." Oxford Studies in Early Modern Philosophy, vol. 7, ed. Daniel Garber and Donald Rutherford, 257308. Oxford: Oxford University Press.

Stein, H. 1977. "Some Philosophical Prehistory of General Relativity." In Foundations of SpaceTime Theories, ed. John Earman, Clark N. Glymour, and John J. Stachel, 3-49. Minnesota Studies in the Philosophy of Science 8. Minneapolis: University of Minnesota Press.

Vilain, C. 1996. La mécanique de Christian Huygens. Paris: Blanchard.

Wren, C. 1668. "Lex naturae de collisione corporum.” Philosophical Transactions 4:867-68. 